\title{
Perioperative respiratory complications: current evidence and strategy discussed in 2017 JA symposium
}

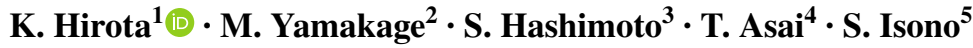

Received: 20 September 2017 / Accepted: 31 October 2017 / Published online: 13 November 2017

(C) Japanese Society of Anesthesiologists 2017

\begin{abstract}
Respiratory management during general anesthesia aims to safely secure the airway and maintain adequate ventilation to deliver oxygen to the vital organs, maintaining homeostasis even during surgery. Despite its clinical importance, anesthesiologists often encounter difficulties in properly managing respiration during the perioperative period, leading to severe respiratory complications. In this year's JA symposium, 5 editorial board members of Journal of Anesthesia (JA) who are experts in the field of respiratory management in anesthesia discussed the following topics: quitting smoking before surgery: exposure to passive smoke is damaging to children, ventilator-associated pneumonia, high inspiratory oxygen concentration and lung injury, aspiration pneumonia, and postoperative respiratory management strategy in patients with obstructive sleep apnea. We hope that this special article regarding this year's JA symposium may be useful for JA readers to manage clinical anesthesia on a daily basis.
\end{abstract}

Keywords Respiration - Airway · Complication . Perioperative management

K. Hirota

hirotak@hirosaki-u.ac.jp

1 Department of Anesthesiology, Hirosaki University Graduate School of Medicine, Hirosaki 036-8562, Japan

2 Department of Anesthesiology, Sapporo Medical University School of Medicine, Sapporo 060-8543, Japan

3 Department of Anesthesiology, Kyoto Prefectural University of Medicine, Kyoto 602-8566, Japan

4 Department of Anesthesiology, Dokkyo Medical University, Koshigaya Hospital, Koshigaya 343-8555, Japan

5 Department of Anesthesiology, Chiba University Graduate School of Medicine, Chiba 263-8670, Japan

\section{Introduction}

Respiratory management during general anesthesia aims to safely secure the airway and maintain adequate ventilation for delivering oxygen to the vital organs, maintaining homeostasis even during surgery. Despite its clinical importance, anesthesiologists often encounter difficulties in properly managing respiration during the perioperative period, leading to severe respiratory complications. In this year's JA symposium held on Friday, June 9 at the JSA Annual Meeting 2017, 5 editorial board members of Journal of Anesthesia (JA)--Drs. Yamakage (Sapporo), Hashimoto (Kyoto), Hirota (Hirosaki), Asai (Koshigaya) and Isono (Chiba)—discussed perioperative respiratory complications associated with smoking, ventilation, high inspired oxygen concentration, aspiration and obstructive sleep apnea syndrome, respectively.

\section{Quitting smoking before surgery: exposure to passive smoke is damaging to children (Yamakage)}

It is a fact that preoperative smoking increases the risk of not only general postoperative complications, but also woundrelated complications, infection, respiratory and neurologic complications, and intensive care unit (ICU) admission. In particular, the risk of respiratory complications is $73 \%$ higher in preoperative smokers than in non-smokers [relative risk 1.73; 95\% confidence interval (CI) 1.35-2.23] [1]. In addition, bone healing has been shown to be better with a longer period of smoking cessation before an operation [2]. In this section, the effects of passive smoking exposure on children are emphasized. Of the smoke inhaled during passive smoking, $15 \%$ is exhaled from the lungs of the smoker; 
this mainstream smoke contains hazardous substances that have been somewhat reduced by the filter. The remaining $85 \%$ is emitted from the burning tip of the cigarette and contains unfiltered hazardous substances and is called secondhand smoke, which is passively inhaled [3]. Numerous studies have pointed out that when children exposed to passive smoking undergo surgery, they suffer from higher rates of airway and respiratory complications [4-6]. Chiswell and Akram [7] performed a systematic review and meta-analysis to determine the impact of environmental tobacco smoke (ETS) exposure on anaesthetic, intraoperative and postoperative harm in children. They found that ETS exposure significantly increased the risk of perianesthetic respiratory adverse events (pooled risk ratio 2.52, 95\% CI 1.68-3.77). Subgroup analysis regarding laryngospasm showed a significant increase by ETS with a risk ratio of 3.54 (95\% CI 2.37-5.28, $p<0.001)$. Interestingly, Lakshmipathy et al. [8] reported that if only one parent smoked, the rate of laryngospasm was higher if the smoker was the mother, rather than the father. Although the researchers were unable to investigate the actual amount of time spent in contact with the children, it can be easily understood that mothers spend more time with their children than fathers do, and that the opportunities for passive smoking are correspondingly higher. Lyons et al. [6] similarly investigated and reported that children exposed to passive smoking exhibited a higher frequency of desaturation in the postoperative recovery room. Interestingly, the more cigarettes their parents smoked per day, the more likely children were to develop desaturation (Table 1). In 1999, the World Health Organization estimated that approximately $50 \%$ of children worldwide were exposed to passive smoking [4]. Many studies have shown that passive smoking is damaging to children's health and, as described above, that there is no reason to doubt the adverse effect of passive smoking on children undergoing surgery. It is our fervent hope to live in a smoke-free society and that symposia on the health hazards of smoking to both the smoker and the exposed children will no longer be needed.

Table 1 Relationship between daily cumulative number of cigarettes smoked per household per day and incidence of desaturation $\left(\mathrm{SaO}_{2}<95 \%\right)$

\begin{tabular}{cccrrr}
\hline \multicolumn{5}{c}{ Number of cigarettes smoked/day } \\
\cline { 2 - 6 } & 0 & $<10$ & $10-20$ & $20-40$ & $>40$ \\
\hline Desaturation episode & & & & \\
$(-)$ & $55(89 \%)$ & $10(91 \%)$ & $14(74 \%)$ & $17(68 \%)$ & $3(50 \%)$ \\
$(+)$ & $7(11 \%)$ & $1(9 \%)$ & $5(26 \%)$ & $8(32 \%)$ & $3(50 \%)$ \\
\hline
\end{tabular}

The cumulative total number (\%) of cigarettes smoked by those to whom the child was exposed was also related to desaturation $(p<0.005)$. Table 1 was taken from Reference [6]

\section{Ventilator-associated pneumonia (Hashimoto)}

Ventilator-associated pneumonia (VAP) is a type of nosocomial pneumonia that emerges 48 or more hours after mechanical ventilation. In the perioperative period, it can occur postoperatively in patients receiving major surgery or having serious morbidity or complications that require a longer period of ventilatory support. Once occurred, attributable mortality varies between studies, from 0 to $69 \%$ depending on patients' background [9]. In particular, surgical patients with higher clinical prognostic and severity score show high mortality, with incremental economic costs [10]. Most criteria or definitions for VAP diagnosis are not very sensitive or specific, and there is considerable inter-observer variation. About one-third of patients diagnosed with VAP did not have any evidence of pneumonia. Thus, the incidence of VAP also varies between studies: $0-22.2$ cases per 1000 ventilator-days $[10,11]$. In 2013, the Centers for Disease Control and Prevention (CDC) started surveillance for ventilator-associated events (VAE), which included VAP, sepsis, ARDS, pulmonary embolism, barotrauma, and pulmonary edema. However, no study has properly validated this method of diagnosing VAP yet. Multidrug resistant organisms such as Pseudomonas aeruginosa or Acinetobacter baumannii are now leading causes of VAP globally except in Japan and a limited number of other developed countries $[12,13]$. Once diagnosed, appropriate initial empirical therapy with broad-spectrum antibiotics is the key to treating VAP, together with subsequent de-escalation upon identification of the causative orgasms [11]. Prevention strategies have been proposed from several societies. VAP prevention bundles by the Japanese Society of Intensive Care [14] are as follows: do practice secure hand hygiene, do not change ventilator circuit routinely, do deliver appropriate sedation avoiding deep sedation, do daily spontaneous breathing trial to facilitate weaning from ventilatory support. These antimicrobial and bundle strategies are believed to decrease the incidence and mortality of VAP but implementation of these strategies is not always easy.

\section{High inspiratory oxygen concentration and lung injury (Hirota)}

Why do we use high inspiratory oxygen concentration during general anesthesia? It may be due to prevention of hypoxemia. The time to a decrease in $\mathrm{SpO}_{2}$ to less than $90 \%$ has been reported to be 411,303 and 213 s following apnea with preoxygenation with 100,80 and $60 \%$ oxygen, respectively [15]. In an unexpected "cannot ventilate, cannot intubate" case, you may have about 7 min until the patient is hypoxic when you have sufficiently performed preoxygenation with $100 \%$ oxygen. During 7 min, you can give 
sugamadex and naloxone with termination of propofol infusion and may consequently save the patient's life. Thus, use of high inspired oxygen concentrations $\left(\mathrm{FiO}_{2}\right)$ has a strong logical merit for induction of general anesthesia. In addition, intraoperative high $\mathrm{FiO}_{2}$ has been reported to reduce the incidence of surgical site infection and postoperative nausea and vomiting [16]. However, high $\mathrm{FiO}_{2}$ can also cause several adverse reactions such as absorption atelectasis [15], acute exacerbation of idiopathic pulmonary fibrosis [17] and VAP [18]. There is evidence that $100 \%$ oxygen is a major cause of absorption atelectasis during general anesthesia, as $100 \%$ oxygen washes out nitrogen from alveoli and fills the alveoli. In contrast to nitrogen, oxygen quickly diffuses into the pulmonary vasculature and then dissolves into the blood. Consequently, gas left in the alveoli is insufficient to prevent the alveolus collapsing [15]. Intraoperative high $\mathrm{FiO}_{2}$ is also thought to cause acute exacerbation of idiopathic pulmonary fibrosis after lung surgery with one-lung ventilation-induced ischemic/reperfusion injury. Some patients show honeycomb findings in the subpleural space (within $1 \mathrm{~cm}$ from pleura), defined as localized interstitial pneumonitis findings by chest computerized tomography. These patients may have a high risk of postoperative acute exacerbation of idiopathic pulmonary fibrosis even if they do not reveal any abnormal findings in the pulmonary functional test [17]. Hyperoxemia often occurs in mechanically ventilated patients with high $\mathrm{FiO}_{2}$ in the ICU [18]. Although high $\mathrm{FiO}_{2}$ may prevent hypoxia and improve the tissue oxygen supply, hyperoxemia may also induce vasoconstriction and decrease cardiac output, resulting in reduced blood flow [18]. In addition, hyperoxia is responsible for hyperoxic acute lung injury, revealing pulmonary edema, hyaline membrane formation, pulmonary arteriole thickening, and alteration in the ventilation/perfusion fraction [18]. High $\mathrm{FiO}_{2}$ inhibits surfactant production to promote pulmonary atelectasis, and impairs mucociliary clearance and immune function of immune cells [18]. These are known to be risk factors of VAP. Indeed, it has been reported that hyperoxemia is independently associated with VAP [18]. A recent study [19] reports that a conservative oxygenation strategy (targeted $\mathrm{SpO}_{2}: 88-92 \%$ ) appears not to be inferior to liberal oxygenation $\left(\mathrm{SpO}_{2} \geq 96 \%\right)$ in mechanically ventilated patients. Therefore, we should carefully use or avoid perioperative high $\mathrm{FiO}_{2}$.

\section{Aspiration pneumonia (Asai)}

The danger of pulmonary aspiration during general anesthesia was recognized in the 1930 s, mainly by obstetric surgeons [20]. Since then, much effort has been made to minimize aspiration. This includes preoperative fasting, the use of a cuffed tracheal tube, and in patients at increased risk of aspiration, preoperative use of apomorphine, and suction of gastric contents through a gastric tube. In the 1950 s, rapid-sequence induction of anesthesia was developed [21], but pulmonary aspiration of gastric contents frequently occurred. In the 1960s, cricoid pressure was found to be effective in preventing aspiration during rapid-sequence induction of anesthesia [22], and the induction method was established in the 1970s [23]. With these efforts, the incidence and severity of pulmonary aspiration appears to be decreased. Nevertheless, a recent large-scale prospective observational study in Britain has shown that pulmonary aspiration is still the main cause of death or life-threatening airway complications [24]. This survey also indicated that roughly $50 \%$ of cases of aspiration occurred during the use of a supraglottic airway, which would not be used in patients at high risk of aspiration [25]. Therefore, we need to make further efforts to reduce the incidence of pulmonary aspiration during and after anesthesia [25], and to find better methods of airway management (Fig. 1).

\section{Postoperative respiratory management strategy in patients with obstructive sleep apnea (Isono)}

Obstructive sleep apnea (OSA) is a common disease in the general adult population as well as the surgical population [26]. Preoperative OSA screening is effectively performed using a questionnaire and a portable sleep monitor, enabling individual perioperative airway management [26]. Both mask ventilation and tracheal intubation during anesthesia induction are more difficult in OSA patients [27], and the JSA airway management guideline is helpful to prepare and manage airway difficulties [28]. OSA is also an independent risk factor for postoperative cardiorespiratory complications [26]. Pre-existing OSA worsens postoperatively with the peak increase in apnea-hypopnea index on postoperative night 3 [26]. While postoperative changes in sleep architecture, lung volume reduction, upper airway edema formation



Fig. 1 Incidence of laryngospasm in passive smoking exposure. Relative risk for passive smoking exposure and laryngospasm $=10.0$ (95\% CI 2.2-45.6; $p<0.001$ ) Figure 1 was taken and modified from Reference [8] 
and use of opioids would be the candidates for postoperative OSA worsening, the mechanisms have not been clarified. Opioids are strong depressants of the upper airway motoneurons, possibly worsening OSA and even resulting in "dead in bed" [26]. Furthermore, the increased sensitivity to opioids recently documented in both OSA children and adults makes the situation worse and complicated [26]. Accordingly, establishment of upper airway maintenance strategies immediately after surgery and during the postoperative nights is of great clinical importance for the anesthesiologist [29]. The nasal continuous positive pressure is the first choice for OSA treatment [26]. However, its acceptance rate may be limited in newly-diagnosed OSA patients undergoing surgery [26], suggesting the necessity of alternative postoperative OSA treatment protocols. Oxygen therapy is beneficial for maintaining oxygenation and reduction of OSA frequency, particularly in patients with abnormally higher responsiveness to respiratory chemo-stimuli [26]. Insertion of a nasal airway does not completely eliminate OSA and decreases the OSA frequency by only half, and it is probably impractical to use the painful wide-bore tube routinely [30]. Nasal high-flow oxygen therapy may be useful for patients with preoperatively undiagnosed moderate to severe OSA since the airway pressure is expected to increase by approximately $1 \mathrm{cmH}_{2} \mathrm{O}$ with each $10 \mathrm{~L} / \mathrm{min}$ increase in flow rate [31]. In our experience, the acceptance rate is much higher compared with nasal CPAP therapy. It should be noted that none of the alternative treatments to nasal CPAP are sufficient to fully dilate the pharyngeal airway and therefore they need to be combined with other alternatives to normalize the pharyngeal closing pressure. Increasing the airway pressure by $8 \mathrm{cmH}_{2} \mathrm{O}$ above the pharyngeal closing pressure normalizes breathing. Therefore, even without CPAP treatment, any combination of the alternative treatments achieving $8 \mathrm{cmH}_{2} \mathrm{O}$ reduction of the closing pressure in total should have a similar effect to CPAP treatment and be able to improve breathing during sleep [32]. The magnitude of pharyngeal closing pressure reduction by each airway management technique is illustrated in Fig. 2. For example, a combination of the use of a pillow (sniffing position) and sitting position $\left(60^{\circ}\right.$ headup posture) is expected to reduce the closing pressure by $9 \mathrm{cmH}_{2} \mathrm{O}\left(3 \mathrm{cmH}_{2} \mathrm{O}+6 \mathrm{cmH}_{2} \mathrm{O}\right)$ [33]. In practice, a semi-sitting position $\left(30^{\circ}\right.$ head-up posture) with the use of a pillow is advantageous for maintaining both airway patency and lung volume in most postoperative patients. In addition, the further addition of $30 \mathrm{~L} / \mathrm{min}$ high-flow nasal oxygen therapy, which is expected to decrease the closing pressure by $3 \mathrm{cmH}_{2} \mathrm{O}$, would further increase the effectiveness in these positions and may help to open airways in patients with severe OSA. Mouth opening should be prevented because mouth opening per se increases the closing pressure by $3 \mathrm{cmH}_{2} \mathrm{O}$ and also reduces the effectiveness of nasal CPAP and high-flow nasal oxygen therapy by introducing massive

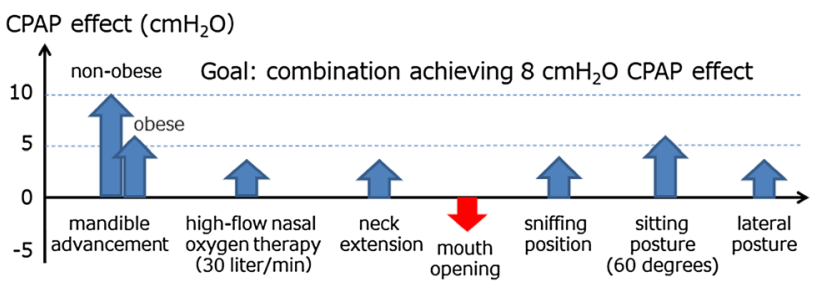

Fig. 2 Effectiveness of various airway interventions for maintaining upper airway patency. Combinations of airway interventions achieving $8 \mathrm{cmH}_{2} \mathrm{O}$ CPAP effect could improve breathing during sleep while the responses may differ individually

air leakage through the mouth. Responses to the alternative treatments significantly differ between patients, and therefore the combination strategy should be individualized based on proper respiratory monitoring.

\section{Conclusion}

In this symposium, we discussed perioperative respiratory complications and their preventive strategies. We hope that this symposium may be useful for you to manage clinical anesthesia on a daily basis.

\section{References}

1. Grønkjær M, Eliasen M, Skov-Ettrup LS, Tolstrup JS, Christiansen AH, Mikkelsen SS, Becker U, Flensborg-Madsen T. Preoperative smoking status and postoperative complications-a systematic review and meta-analysis. Ann Surg. 2014;259:52-71

2. Truntzer J, Vopat B, Feldstein M, Matityahu A. Smoking cessation and bone healing: optimal cessation timing. Eur J Orthop Surg Traumatol. 2015;25:211-5.

3. Henderson AJ. The effects of tobacco smoke exposure on respiratory health in school-aged children. Paediatr Respir Rev. 2008;9:21-8.

4. O'Rourke JM, Kalish LA, McDaniel S, Lyons B. The effects of exposure to environmental tobacco smoke on pulmonary function in children undergoing anesthesia for minor surgery. Pediatr Anesth. 2006;16:560-7.

5. Skolnick ET, Vomvolakis MA, Buck KA, Mannino SF, Sun LS. Exposure to environmental tobacco smoke and the risk of adverse respiratory events in children receiving general anesthesia. Anesthesiology. 1998;88:1144-53

6. Lyons B, Frizelle H, Kirby F, Casey W. The effect of passive smoking on the incidence of airway complications in children undergoing general anesthesia. Anaesthesia. 1996;51:324-6.

7. Chiswell C, Akram Y. Impact of environmental tobacco smoke exposure on anaesthetic and surgical outcomes in children: a systematic review and meta-analysis. Arch Dis Child. 2017;102:123-30.

8. Lakshmipathy N, Bokesch PM, Cowen DE, Lisman SR, Schmid $\mathrm{CH}$. Environmental tobacco smoke: a risk factor for pediatric laryngospasm. Anesth Analg. 1996;82:724-7 
9. Melsen WG, Rovers MM, Bonten MJ. Ventilator-associated pneumonia and mortality: a systematic review of observational studies. Crit Care Med. 2009;37:2709-18.

10. Hortal J, Giannella M, Pérez MJ, Barrio JM, Desco M, Bouza E, Muñoz P. Incidence and risk factors for ventilator-associated pneumonia after major heart surgery. Intensive Care Med. 2009;35:1518-25

11. Nair GB, Niederman MS. Ventilator-associated pneumonia: present understanding and ongoing debates. Intensive Care Med. 2015;41:34-48.

12. Annual open report 2015. Japan nosocomial infections surveillance. https://janis.mhlw.go.jp/english/report/open_ report/2015/4/1/ken_Open_Report_Eng_201500_clsi2012.pdf. Accessed 11 Oct 2017.

13. Annual open report 2016 from ICU, Japan nosocomial infections surveillance (in Japanese). https://janis.mhlw.go.jp/report/open_ report/2016/3/3/ICU_Open_Report_201600.pdf. Accessed 11 Oct 2017.

14. Public report of VAP bundle from Japanese Society of Intensive Care Medicine (in Japanese). http://www.jsicm.org/pdf/2010VAP. pdf.

15. Edmark L, Kostova-Aherdan K, Enlund M, Hedenstierna G. Optimal oxygen concentration during induction of general anesthesia. Anesthesiology. 2003;98:28-33.

16. Hovaguimian F, Lysakowski C, Elia N, Tramèr MR. Effect of intraoperative high inspired oxygen fraction on surgical site infection, postoperative nausea and vomiting, and pulmonary function: systematic review and meta-analysis of randomized controlled trials. Anesthesiology. 2013;119:303-16.

17. Kondoh Y, Taniguchi H, Kitaichi M, Yokoi T, Johkoh T, Oishi T, Kimura T, Nishiyama O, Kato K, du Bois RM. Acute exacerbation of interstitial pneumonia following surgical lung biopsy. Respir Med. 2006;100:1753-9.

18. Six S, Jaffal K, Ledoux G, Jaillette E, Wallet F, Nseir S. Hyperoxemia as a risk factor for ventilator-associated pneumonia. Crit Care. 2016;20:195.

19. Panwar R, Hardie M, Bellomo R, Barrot L, Eastwood GM, Young PJ, Capellier G, Harrigan PW, Bailey M, CLOSE Study Investigators, ANZICS Clinical Trials Group. Conservative versus liberal oxygenation targets for mechanically ventilated patients. A pilot multicenter randomized controlled trial. Am J Respir Crit Care Med. 2016;193:43-51.

20. Asai T, Shingu K. Should Mendelson's syndrome be renamed? Anaesthesia. 2001;56:398-9.
21. Morton HJ, Wylie WD. Anaesthetic deaths due to regurgitation or vomiting. Anaesthesia. 1951;6:190-201.

22. Sellick BA. Cricoid pressure to control regurgitation of stomach contents during induction of anaesthesia. Lancet. 1961;2:404-6.

23. Stept WJ, Safar P. Rapid induction-intubation for prevention of gastric-content aspiration. Anesth Analg. 1970;49:633-6.

24. Cook TM, Woodall N, Frerk C. Major complications of airway management in the UK: results of the 4th National Audit Project of the Royal College of Anaesthetists and the Difficult Airway Society. Part 1 anaesthesia. Br J Anaesth. 2011;106:617-31.

25. Asai T. Who is at increased risk of pulmonary aspiration? (Editorial). Br J Anaesth. 2004;93:497-500.

26. Liao P, Wong J, Singh M, Wong DT, Islam S, Andrawes M, Shapiro CM, White DP, Chung F. Postoperative oxygen therapy in patients with OSA: a randomized controlled trial. Chest. 2017;151:597-611.

27. Sato S, Hasegawa M, Okuyama M, Okazaki J, Kitamura Y, Sato Y, Ishikawa T, Sato Y, Isono S. Mask ventilation during induction of general anesthesia: influences of obstructive sleep apnea. Anesthesiology. 2017;126:28-38.

28. Japanese Society of Anesthesiologists. JSA airway management guideline 2014: to improve the safety of induction of anesthesia. J Anesth. 2014;28:482-93.

29. Isono S. Obstructive sleep apnea of obese adults: pathophysiology and perioperative airway management. Anesthesiology. 2009;110:908-21.

30. Kumar AR, Guilleminault C, Certal V, Li D, Capasso R, Camacho M. Nasopharyngeal airway stenting devices for obstructive sleep apnoea: a systematic review and meta-analysis. J Laryngol Otol. 2015;129:2-10.

31. Parke RL, Eccleston ML, McGuinness SP. The effects of flow on airway pressure during nasal high-flow oxygen therapy. Respir Care. 2011;56:1151-5.

32. Isono S. Optimal combination of head, mandible and body positions for pharyngeal airway maintenance during perioperative period: lesson from pharyngeal closing pressures. Semin Anesth. 2007;26:83-93.

33. Tagaito $\mathrm{Y}$, Isono $\mathrm{S}$, Tanaka A, Ishikawa T, Nishino T. Sitting posture decreases collapsibility of the passive pharynx in anesthetized paralyzed patients with obstructive sleep apnea. Anesthesiology. 2010;113:812-8. 